\title{
Dynamic pattern generation by singlemode fibers for endoscopic 3D measurement systems
}

Pulwer, Silvio, Jahns, Daniel, Villringer, Claus, Schrader, Sigurd

Silvio Pulwer, Daniel Jahns, Claus Villringer, Sigurd Schrader, "Dynamic pattern generation by singlemode fibers for endoscopic 3D measurement systems," Proc. SPIE 11293, MOEMS and Miniaturized Systems XIX, 112930F (28 February 2020); doi: 10.1117/12.2543526

SPIE. Event: SPIE OPTO, 2020, San Francisco, California, United States 


\title{
Dynamic pattern generation by singlemode fibers for endoscopic 3D measurement systems
}

\author{
Silvio Pulwer*a, Daniel Jahns ${ }^{\mathrm{a}, \mathrm{b}}$, Claus Villringer ${ }^{\mathrm{a}}$, Sigurd Schrader ${ }^{\mathrm{a}}$ \\ ${ }^{a}$ Technical University of Applied Sciences Wildau, Hochschulring 1, 15745 Wildau, Germany \\ bINDI-Jahns, Industriestrasse 1-3, 12099 Berlin, Germany
}

\begin{abstract}
This work presents experimental and simulation results of a 3D measurement concept based on fiber-optic interference pattern generation. A demonstrator system which is able to adjust different optical and mechanical parameters is described. The results of parameter variation studies are discussed along with possible applications in the field of micro-optical 3D measurement systems like endoscopes. The experiments were prepared with different structures with various scattering properties. To implement that approach into a micro-optical 3D measurement system, the combination of distance variation between the fibers and a controlled phase shift introduced by a retarder can be used to design the system versatile.
\end{abstract}

Keywords: Endoscope, 3D Measurement, Phase Shift, Fiber

\section{INTRODUCTION}

The precise measurement of surfaces in difficult accessible areas, is one of the key elements for an efficient and reliable production. A miniaturized visible inspection device, is able to detect smallest surface damages in complex machines without demounting it. Hence it serves for early detection of damages and helps to develop the maintaining procedure more efficient. In particular, endoscopic systems have the potential to shrink in overall size and add features like 3D measurement techniques by optimizing the sub-systems containing different types of opto-mechanical components.

Companies that offer triangulation based 3D endoscopes use different approaches including phase shifting techniques. The pattern generator includes diffractive optical elements or projection optics. All systems have common problems that are based on the fact, that the obtained wrapped phase map contains true phase discontinuities which are originating from the object surface discontinuities. Therefore it is essential to overcome this problem by phase unwrapping strategies based on multiple phase maps generated by varying the spatial frequency of the projected fringe patterns [1]. In addition to that, coded phase shifting techniques as described by Wissmann et al. [2] can reduce the total acquisition time by effectively reducing the number of pattern projections.

\section{MOTIVATION}

In this work, we will focus on an independent sub-system for generating dynamic patterns to use it for triangulation based 3D measurement evaluations. Hereby the range of features of available endoscopic systems can be extended and enable more reliable and more stable measurements. Therefore the system has certain requirements that cover mechanical and optical properties as well as resulting constraints. For the triangulation measurement, a certain distance between the subsystem of the camera and the projection sub-system is necessary. It defines, depending on the working distance between the distal end of the endoscope and the object plane (d), the triangulation angle $(\varphi)$. For a higher depth sensitivity it is crucial to increase $(\varphi)$ by for example shrinking down the foot print of each individual sub-systems. To guarantee the flexibility and maneuverability of the whole endoscopic system, rigid opto-mechanical elements between the endoscope distal tip and the proximal end should be avoided. Furthermore, the length of the tip should be as short as possible to reach a low bending radius. By introducing new opto-mechanical features that support the 3D measurement process, it is possible to overcome problems like a limited depth of field or vignetting effects. Solutions for that could be the implementation of an electrically tunable lens in the imaging system including aperture control mechanics [3].

* pulwer@th-wildau.de; phone: +49 3375508771; www.th-wildau.de/photonik

MOEMS and Miniaturized Systems XIX, edited by Wibool Piyawattanametha, Yong-Hwa Park, Hans Zappe, Proc. of SPIE Vol. 11293, 112930F · (c) 2020 SPIE · CCC code: 0277-786X/20/\$21 · doi: 10.1117/12.2543526 


\section{SETUP AND RESULTS}

The proposed pattern generating sub-system is based on fiber-optic interference as described in the following.

The radiation of two (or more) neighbored singlemode fibers, whose behavior is close to a Gaussian beam (TEM00-Mode), creates due to interference effects, a line pattern inside the overlapping volume. Hereby, the spatial frequency of the fringe pattern at the object plane depends on the distance between each individual optical fiber $(\delta)$, the wavelength $(\lambda)$ of the coupled light source and the distance to the object plane (d).

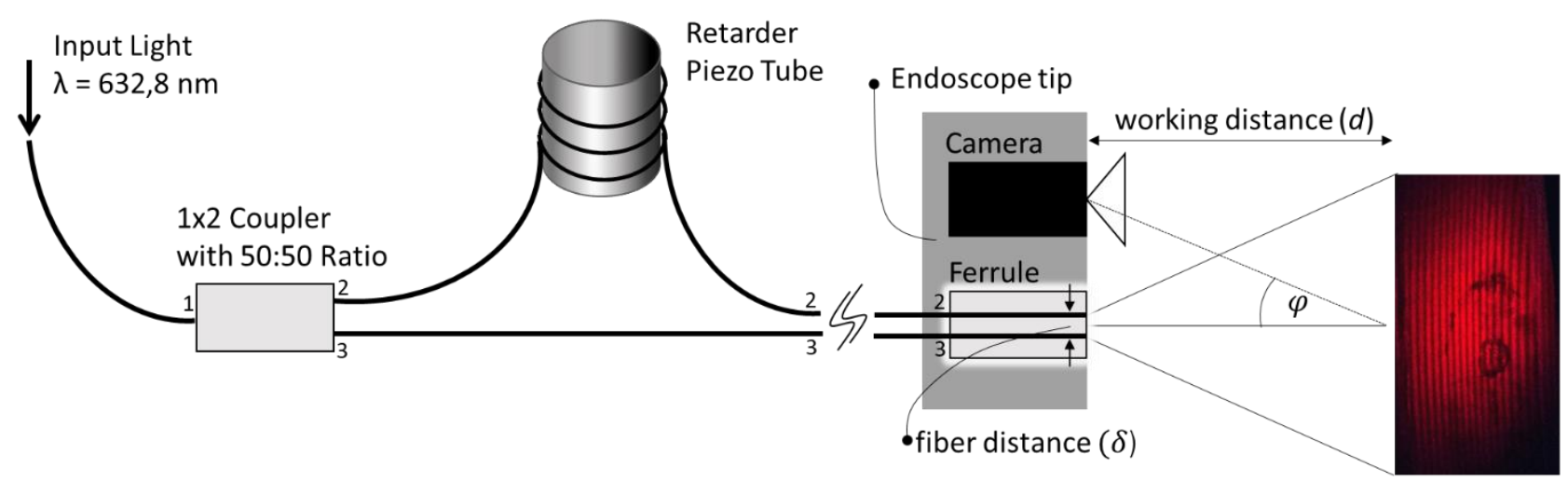

Figure 1. Setup for the fiber-optic interference pattern generator.

In comparison to classical optical projection techniques there are theoretically no limitations for the depth of focus for the generated fringe pattern. Each fiber end acts as a point source and consequently it emits a spherical wavefront with a corresponding wavelength. A pair of fibers emit a bundle of wavefronts with slightly different propagation vectors which will interfere. The generated line patterns are therefore basically Young's fringes [4] and can be seen as parallel fringes with a distance described by

$$
\Delta y=\lambda d / \delta .
$$

In general, the intensity distribution of the projected pattern onto the object plane and captured by a camera positioned at a triangulation angle $(\varphi)$ can be described as

$$
I(x, y)=\cos \left\{\frac{2 \pi \delta}{\lambda d}\left[x \cos \varphi+z_{0}(x, y) \sin \varphi\right]\right\} .
$$

The basic setup contains a coherent He-Ne Laser with $\lambda=632.8 \mathrm{~nm}$ with typical output power of $5 \mathrm{~mW}$, a $2 \times 1$ fiber-optic coupler with 50:50 coupling ratio and a 1 megapixel camera. The fibers are standard HP630 singlemode fibers with $125 \mu \mathrm{m}$ cladding and the ends of the fibers are stripped and cleaved separately.

The main features of the pattern generator are the control over the phase shift, spatial frequency and pattern orientation. Introducing an optical phase shift in one of the fibers is a well-known method to reach a lateral phase shift of the fringe on the object plane pattern [5]. Accordingly one fiber is winded around a voltage applied piezo ceramic tube (lead zirconate titanate) with an inner and outer electrode. As a result, the fiber gets mechanically stretched and depending on the windings, an optical phase shift is introduced. In a first test (Fig. 2) we achieved random phase ( $\Phi$ ) shifts by applying voltage to the piezo ceramic tube.
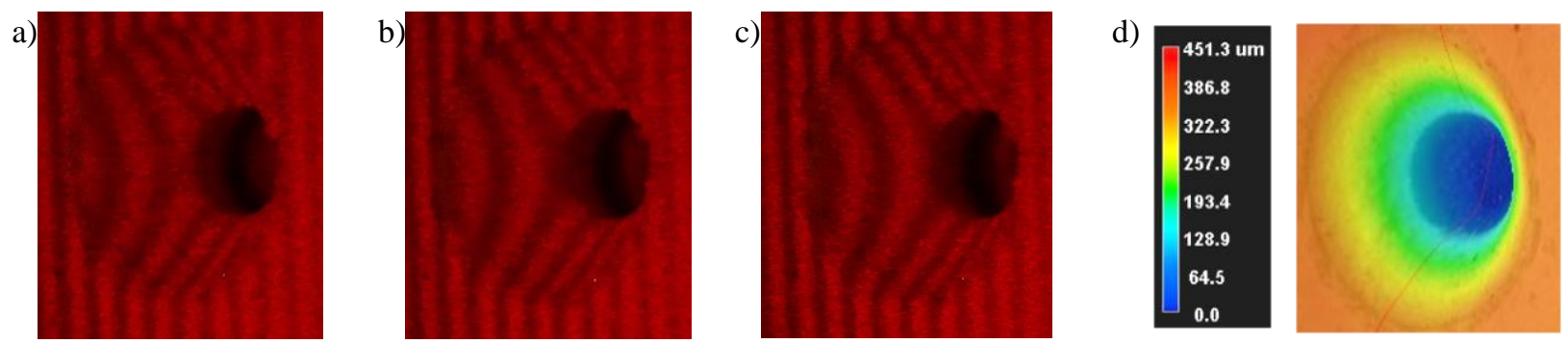

Figure 2. Images (a-c) acquired with fiber-optic interference pattern generator setup at different phase shift values. Sample is a ceramic surface with central valley structure. Image (d) is a 3D reconstruction with colored profile map acquired with Keyence 3D microscope. 
In a next step, the imaged fringe pattern must be translated to a phase map. Here phase shifting techniques [6] as well as Fourier transform analysis [7] can be used. Moreover it requires constant conditions, while multiple fringe patterns are captured by the camera. Duan Xiao-jie et al. showed a method to use this setup with an additional feedback loop for measuring and controlling the phase stability and hence actively reduce noise by stabilizing output phase condition. [4] Additionally the integration of a bundled singlemode fiber array responds to a variable spatial frequency of the obtained fringe pattern by a discrete level, corresponding to the distance of each fiber core. This is fully compatible to extended phase unwrapping methods which require two or more different fringe periods. This method, is suitable especially for rough surfaces where the true phase perhaps changes more than $\pi$ radians in between two neighbored pixel [8].

For the simulation models calculated with Zemax we assume a working distance of $7 \mathrm{~mm}$ to the object plane, a fiber distance of $125 \mu \mathrm{m}$ and a core diameter of $6 \mu \mathrm{m}$ with numerical aperture (NA) $=0.11$. As shown in Fig. 3(a) switching between different pairs of fibers inside the array, the alignment as well as the spatial frequency of the pattern can be adjusted. In addition the pattern is projected on a negative spherical dome with a radius of $500 \mu \mathrm{m}$ and recorded with a simulated camera at a triangulation angle of $20^{\circ}$. The influence of object surface roughness was simulated by differentiating between ideal Lambertian (Fig. 3b) and Gaussian (Fig. 3c) scattering behavior.

a)

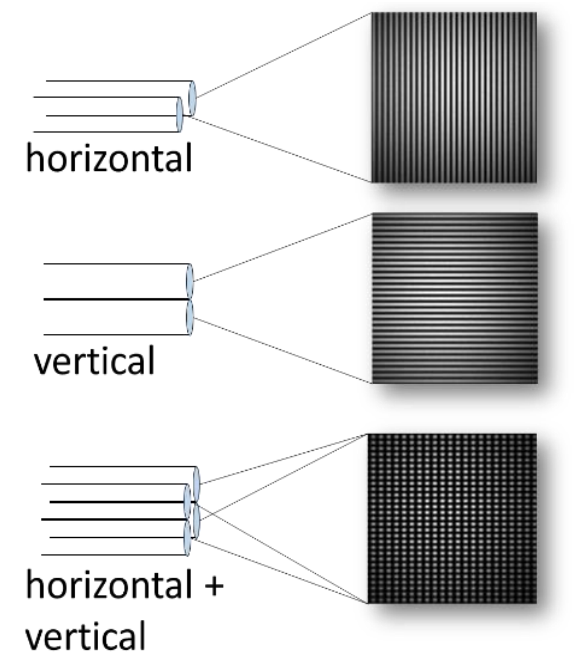

b)

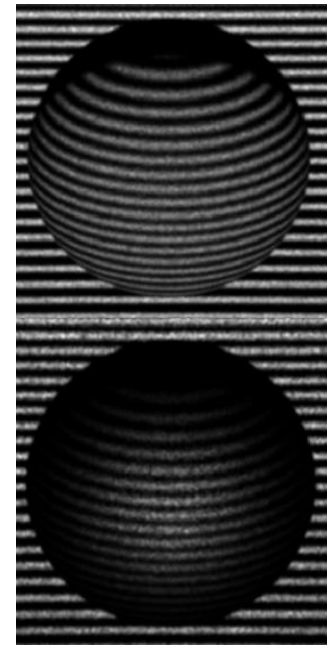

Figure 3. Results of a simulation calculated with Zemax a) of interference fringe patterns caused by different fiber directions; b) of fringe pattern projection on a negative spherical dome with a radius of $500 \mu \mathrm{m}$ and subsequently image acquisition by camera model at triangulation angle of $20^{\circ}$ with Lambertian object surface scattering behavior and c) Gaussian object surface scattering behavior.

Due to speckle and random noise, the captured images should be pre-processed by low-pass filtering and analyzing of the Fourier spectrum. Especially specular reflections and high angle slopes can cause intensity problems which result in not correct detected fringes by the camera. In consequence, higher laser power or improved camera sensitivity are necessary. [5]

In real life cases like the inspection of turbine engine, in particular the combustion chamber and high pressure compressor, materials like titan alloys and high strength steel exhibit similar optical scattering behavior and therefore similar critical measurement conditions. Thus, the diffuse intensity of back scattered light depends on the rms (root mean square) roughness $(\sigma)$, roughness exponent $(h)$ and the lateral correlation length $(\xi)$. The height-height correlation function $\mathrm{C}(\mathrm{x}, \mathrm{y})$ is related to all parameter mentioned before and describes the height fluctuations of the sample surface. [9]

For investigating the impact of $\mathrm{C}(\mathrm{x}, \mathrm{y})$ to the measurement we prepared samples with different correlation length and rms roughness. Therefore, ceramic as well as titan alloy materials were used. The results shown in Fig. 4 demonstrate the influence of the lateral correlation length on the measurability of the samples. This clearly corresponds to different portion of specular reflective characteristics and can cause intensity problems and as well as low true phase contrast. In the case of titan alloy, a system with two or more spatial frequencies would improve phase mapping results. 


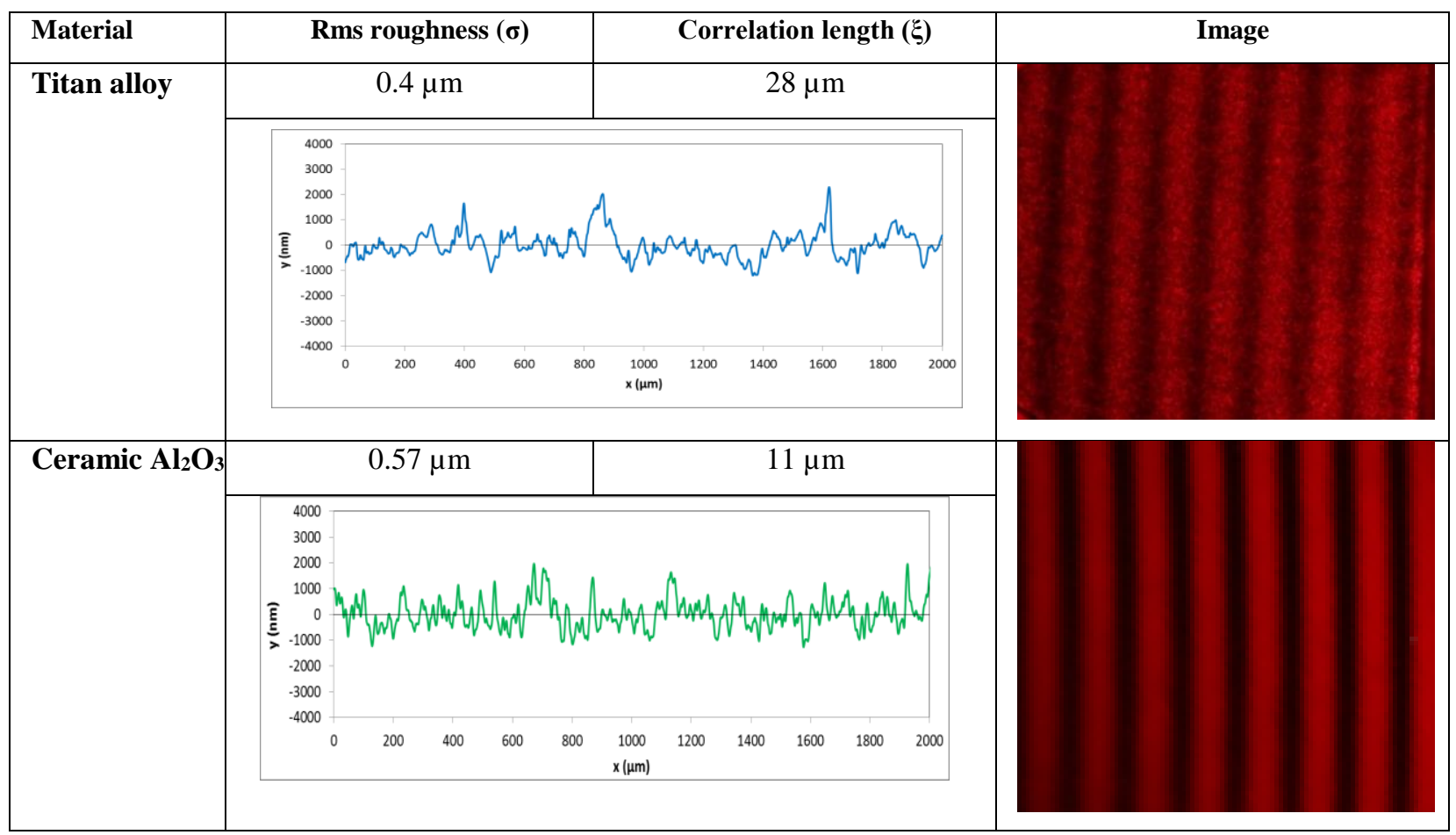

Figure 4. Results of roughness investigations and the impact to the phase contrast of the interference fringe pattern.

In further tests we compared simulation results with real measurement scenarios. Here we used calibration standards that were produced by planning tools. For the simulation, we generated a zig zag structure object with $45^{\circ}$ slope angle similar to the calibration standard that was produced by a planning tool. The metallic surface exhibits high specular reflective characteristics.

a)

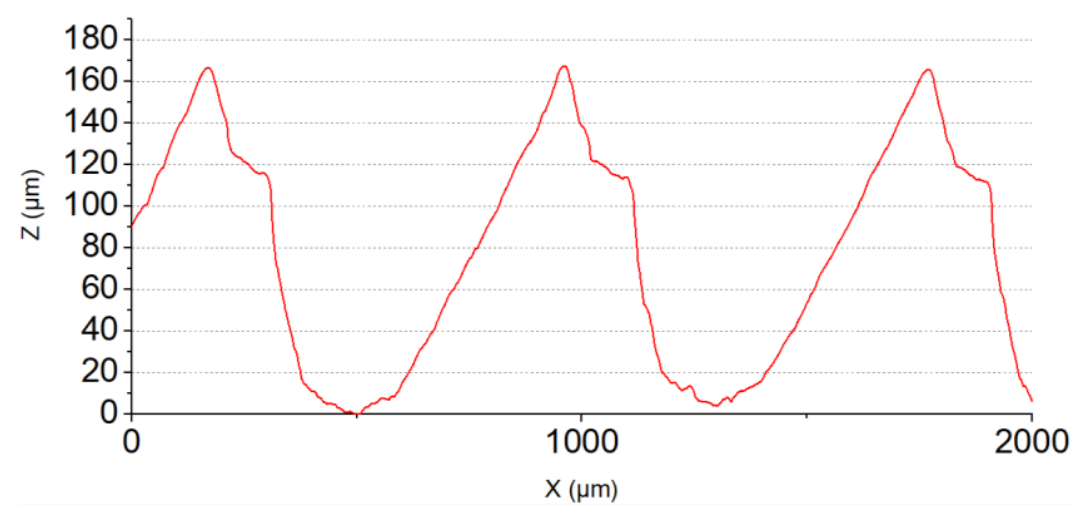

b)

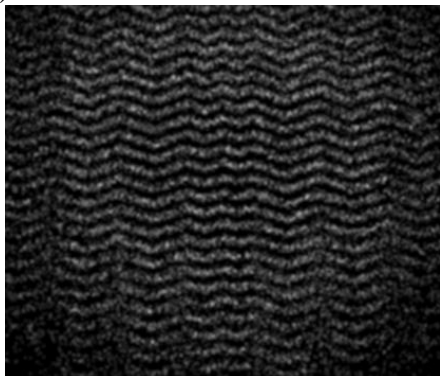

c)

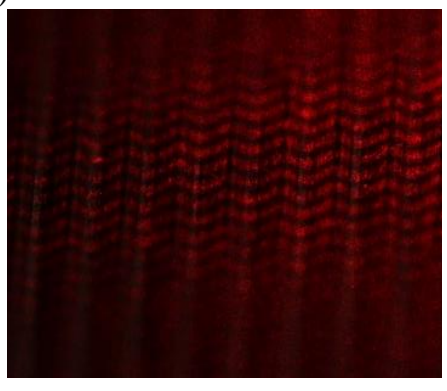

Figure 5. a) Result of tactile profile measurement of calibration test sample. b) Simulation with Zemax of a zig zag structure with Gaussian object surface scattering behavior. c) Real image of test sample acquired with test setup. 


\section{ENDOSCOPIC INTEGRATION}

With the implementation of a small versatile fringe pattern generator into an endoscopic system, standard 2D endoscopes can get additional 3D measurement features. Therefore we focused on keeping the footprint of the generator as small as possible. The concept contains the idea, to integrate the generator into possibly already existing sub-structures like working channels of endoscopes. Thus, a first laboratory setup of the fiber arrangement was based on two singlemode fibers which are mounted and glued close to each other.

In first tests, which are related to a typical endoscopic measurement scenario which means a low working distance, we realized that a standard $125 \mu \mathrm{m}$ single mode fiber setup results in a non-compatible very high spatial frequency for endoscope compatible camera modules. As a consequence, we particularly removed the cladding and coating one side of each fiber. By that, we reduced the distance between the fiber cores and decreased the spatial frequency. Hence the general intensity distribution related to Eq. (2) is compatible to pixel size and resolution of typical endoscope cameras.

As seen in Fig. 6 the fiber is polished over a length of $2.8 \mathrm{~mm}$ to create an acceptable tilt angle in between both fibers. The remaining cladding on the inner side has a thickness between $20 \mu \mathrm{m}$ and $40 \mu \mathrm{m}$ and can be optimized for certain spatial frequencies.
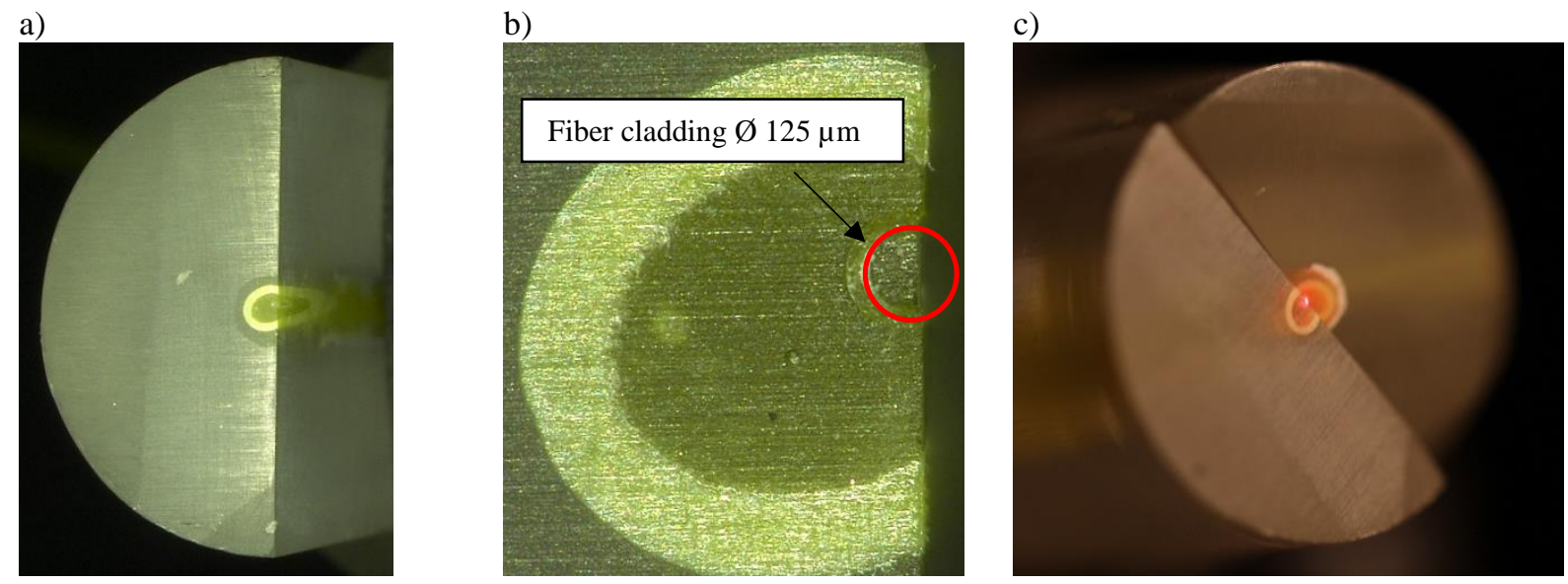

Figure 6. a) Microscope image of the single mode fiber with oblique perspective. b) Microscope cross section image of the fiber with highlighted remaining cladding structure. c) Fibers used within test setup.

To improve the prototyping process, the fiber is attached into a capillary. This capillary can be designed to fit into a standard Ø $3.2 \mathrm{~mm}$ stainless steel SMA905 ferrule which can be inserted into a standard working channel of an endoscope. Because of the remote location of the laser source and phase manipulating devices no further design changes of the tip of the endoscope are necessary.

\section{CONCLUSION}

In this paper, we proposed a 3D measurement concept based on fiber-optic interference pattern generation. To implement that approach into a micro-optical 3D measurement system we used a capillary based fiber mounting approach in combination with a stainless steel ferrule. To guarantee good phase contrast and phase shifting results, simulations that include surface roughness related scattering behavior can support optimizing the phase mapping algorithm. The combination of distance variation between the fibers by inserting singlemode fiber bundles and a controlled phase shift introduced by a retarder can be used to design the system versatile and applicable for many measurement scenarios. This work serves as a first attempt to use this approach for the development of an inexpensive improvement of endoscopic systems. 


\section{ACKNOWLEDGEMENTS}

This project is funded by the Federal Ministry for Economic Affairs and Energy in the frame of the Central Innovation Programme for small and medium-sized enterprises (ZIM) under contract no. ZF4012230WM8.

\section{REFERENCES}

[1] Zappa E, Busca G., "Comparison of eight unwrapping algorithms applied to Fourier-transform profilometry", Optics and Lasers in Engineering, 46(2): 106-16, 2008.

[2] Wissman, P. et al., "Fast and accurate 3D scanning using coded phase shifting and high speed pattern projection", 3DIMPVT- 2011, 2011.

[3] Yongchao Zou et al., "Miniature adjustable-focus endoscope with a solid electrically tunable lens", Optics Express, Vol. 23, Issue 16, pp. 20582-20592, 2015.

[4] Duan Xiao-jie et al., "Phase stabilizing method based on PTAC for fiber-optic interference fringe projection profilometry", Optics \& Laser Technology, 47, 137-143, 2013.

[5] Penning, L. et al., "Miniaturized 3-D surface profilometer using a fiber optic coupler", Optics and Laser Technology 33, 313-320, 2001.

[6] Creath K, "Temporal phase measurement methods", Robinson DW, Reid GT, editors. Interferogram analysis, digital fringe pattern measurement techniques. Bristol: Institute of Physics Publishing, p. 94-140, 1993.

[7] Takeda M, Ina H, Kobayashi S., "Fourier-transform method of fringe-pattern analysis for computer-based topography and interferometry", J Opt Soc Am, 72:156-60, 1982.

[8] Saldner HO, Huntley JM., "Temporal phase unwrapping: application to surface profiling of discontinuous objects", Appl Opt, 36:2770-5, 1997.

[9] Volk, R, "Rauheitsmessung: Theorie und Praxis", Beuth Verlag, 2013 